\title{
An emerging role for chemical biology
}

A lthough significant advances have been made in preventing and treating diseases prevalent in developed countries, including cancer, diabetes and heart disease, there remains an urgent need for enhanced research into diseases that affect millions in developing countries, such as HIV/AIDS, malaria and tuberculosis.

The World Health Organization (http://www.who.org) estimates that malaria, caused by the mosquito-borne parasite Plasmodium falciparum, affects nearly $40 \%$ of the world's population. Tuberculosis, a disease believed to have been conquered in the early twentieth century, has reasserted itself as a major global health threat. The Kaiser Family Foundation (http://www.globalhealthreporting.org) reports that over 2 billion people are infected with Mycobacterium tuberculosis and argues that tuberculosis may kill over 30 million in the coming decade. Both diseases exact a high toll, particularly in the world's most impoverished nations. A sobering fact is that HIV-infected individuals are significantly more susceptible to malaria and tuberculosis. For example, the Global Alliance for TB Drug Development (http://www.tballiance. org) reports that HIV infection increases an individual's risk of contracting tuberculosis by 30 - to 50 -fold and that tuberculosis is the primary cause of death in 30\% of HIV-infected individuals.

Although HIV/AIDS remains a persistent threat, malaria and tuberculosis are curable diseases. However, despite the existence of well-established therapeutic agents, significant difficulties remain in effectively treating these diseases. Tuberculosis treatment is particularly challenging, because current therapies require six to nine months of a strict drug regimen. The development of drug-resistant strains complicates disease prevention and management. Thus, new diagnostic and therapeutic agents will be required, the development of which necessitates a deeper understanding of $P$. falciparum and M. tuberculosis biology.

What can be done? Last year, Richard Klausner and Pedro Alonso outlined several goals of the global fight on malaria (Nature 430, 930-931 (2004)), which included control of mosquito vectors and the development of new preventative and therapeutic drugs. A collaborative initiative called "Grand Challenges in Global Health" (http://www.gcgh.org) has been launched to fund projects with the potential to significantly improve global health. The goals include improving vaccines, identifying strategies to limit drug resistance and developing new diagnostics. In addition, several philanthropic organizations, including the Wellcome Trust (http://www.wellcome.ac.uk), the Burroughs Wellcome Fund (http://www.bwfund.org) and the Bill \& Melinda Gates Foundation (http://www.gatesfoundation.org), have provided early leadership by funding research related to infectious diseases.

How will chemical biology help? Chemical biologists are uniquely positioned to contribute to malaria and tuberculosis research. By studying biological systems at the molecular level, researchers resolve mechanistic questions relating to disease biology. Chemical biologists, adept at creating cross-disciplinary tools, provide fresh insights into the drug discovery process, including target identification, lead candidate optimization and mechanistic studies of drug action. These same approaches may be used to control vectors, develop vaccines and engineer diagnostic systems.

Although DNA-sequencing efforts have provided new windows on malaria and tuberculosis, chemical biology will be instrumental in translating this genetic information into the functional realm. Chemical biologists have rapidly developed methods to profile and analyze diverse proteomes (see Review by Saghatelian \& Cravatt in this issue, p. 130). Comparative proteomics using these tools will illuminate pathogen metabolism and may lead to the identification of unique targets for malaria and tuberculosis drug development.

Chemical biology also offers important mechanistic insights into disease biology. For example, two recent studies highlight the potential of chemical biology to advance M. tuberculosis research. Siderophores are small-molecule ligands that are important for iron acquisition by M. tuberculosis. In this issue, Groves and coworkers (see p. 149 and News \& Views on p. 127) show that siderophores synthesized by $M$. tuberculosis co-opt lipid-trafficking pathways of the host to obtain the iron required for bacterial infectivity. Previously, Quadri, Tan and coworkers (Nature Chemical Biology 1, 29-32 (2005)) identified a key metabolic enzyme involved in siderophore biosynthesis as a new target for M. tuberculosis therapeutic approaches. In addition, we highlight a recent study (see p. 129) that unifies mechanisms of sulfur-fixation pathways, which may represent a new therapeutic target for M. tuberculosis.

Wider socioeconomic issues must be addressed to make diagnosis and treatment options more widely available to those in need. However, chemical biology, with its interdisciplinary focus, can offer the tools and insights necessary to tackle the important societal goal of improving global health. 